

\title{
Impacto de la tecnología en la educación: una evolución en la acción del proceso de enseñanza-aprendizaje
}

\author{
Impact of technology on education: \\ an evolution in the action of the teaching-learning process
}

\section{Sandra Guadalupe Altamirano Galván}

\section{Resumen}

Este trabajo presenta un análisis del impacto que la tecnología ha generado en la educación, provocando grandes cambios que requieren de una adaptación gradual por parte de los actores del proceso, así también para el sistema educativo en el que ha sido necesario hacer ajustes en los métodos de enseñanza, transportar el modelo tradicional hacia el modelo basado en competencias, que era ya urgente para el desarrollo social, sin embargo se hizo aún más presente la necesidad a partir de la intervención de la tecnología y la exigencia del mundo laboral. Esta evolución impacta directamente en el estudiante que debe adaptarse y conocer la responsabilidad que tiene en su aprendizaje, pero también el docente se enfrenta con dos grandes responsabilidades que están inmersas en ese cambio: actualizarse y ayudar al estudiante en su proceso de adaptación, a partir de diseños instruccionales adecuados para ello.

Palabras clave: tecnología, educación, enseñanza, aprendizaje, medios, digitales.

\section{Abstract}

This paper presents an analysis of the impact that technology has generated in education, emerging major changes that require a gradual adaptation by the actors of the process and also for the educational system in which it has been necessary to make variations in the methods education, transport the traditional model to competency-based model, which was now urgent for social development, however, the necessity became even more present after the intervention of technology and the demands of the workplace. This evolution impact directly into the student who must adapt and learn the responsibility of their learning, but also the teacher is faced with two major responsibilities that are embedded in this change: updated and help students in their process of adaptation, from instructional designs suitable.

Keywords: technology, education, teaching, learning, digital, media. 
as nuevas tecnologías y los medios de comunicación en la actualidad, han dado un rumbo diferente a la educación, surgiendo cambios de rol en los actores principales del proceso, que deben adaptarse a pasos agigantados y enfrentarse con sus propios retos y responsabilidades dentro de una sociedad que exige mayor visión y acción ante las diferentes problemáticas del mundo global.

La educación actual se enfrenta con nuevos escenarios que dejan de lado los antiguos paradigmas establecidos, la tecnología desborda sus bondades y las abre al conocimiento, pero el proceso de adaptación no es fácil, surgen ventajas y desventajas.

La llegada de los nuevos medios y su evolución ha sido tan rápida y constante que apenas ha dado tiempo para conocerlos, pero aún no para dominarlos.

Tanto el alumno como el docente, se encuentran en una metamorfosis, ligada a los cambios sociales, surgen nuevas perspectivas y con esto, la necesidad de una visión diferente $y$ una mente abierta al progreso, que demanda madurez tecnológica y el desarrollo de competencias básicas para enfrentarse de lleno con una sociedad que tiene sed de comunicación, calidad e innovación.

La educación actual ha dado un giro a los procesos educativos tradicionales, el alumno es cada vez más autónomo y activo, ya no solo es receptor de información, sino que es capaz de investigar y buscar datos que le sean necesarios en su aprendizaje.
Los nuevos medios de comunicación e información ofrecen al alumno un abanico de posibilidades a la solución de problemas, gracias a la facilidad de interacción con gente de su propio contexto social y de otros contextos, permitiéndole así, ver el mundo desde diferentes perspectivas. Esto debido a que "el usuario ha pasado a tener un papel muy dinámico, a dar a conocer su propia voz, sus ideas y sus obras en la web y a relacionarse con otros usuarios a un nivel sin precedentes en la historia" (Buxarrais y Ovide, 2011, párr.7).

Con esto, podemos decir que, el impacto de los nuevos medios de comunicación en la educación es positivo para el aprendizaje, pero también es importante tomar en cuenta que se debe estar preparado para enfrentar a la tecnología en estos procesos, puesto que puede confundirse la facilidad de acceso a la web y a los nuevos medios tecnológicos, con la facilidad de su aplicación en la educación, y en definitiva, hay una diferencia muy marcada entre estos dos puntos.

"Las nuevas tendencias, dentro del desarrollo de la tecnología educativa, apuntan hacia un incremento de las actividades basadas en la interacción y la creación colectiva de conocimientos" (Avello y Duart, 2016, párr.7). En la actualidad, el mundo requiere de individuos capaces de adaptarse y desarrollarse en contextos y situaciones de cambio constantes. La evolución de la sociedad y las continuas transformaciones a las que nos enfrentamos, nos exigen ser conscientes y partícipes de los procesos de renovación. Somos factores y agentes de cambio, y debemos estar

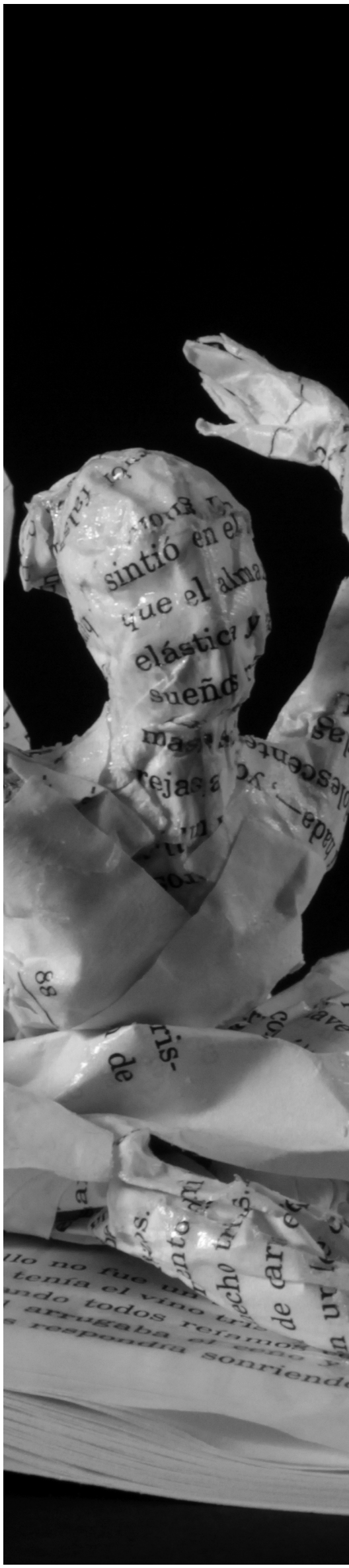


preparados para afrontar tan importante papel."Los cambios sociales, en el entorno laboral y formativo, repercuten sobre el contexto educativo. La velocidad de las respuestas a las necesidades educativas de la sociedad, muestra insuficiencia y lentitud de la reacción de las instituciones responsables" (Bustos, 2005, p.11).

En nuestros tiempos, uno de los objetivos de la educación debería enfocarse en lo que la sociedad requiere con respecto al conocimiento de las nuevas tecnologías, para de esta manera preparar individuos que sean competentes en las exigencias que se les presentarán en la realidad en la que se desenvuelvan.

Es necesario implementar estrategias como la resolución de problemas, pensamiento crítico, razonamiento y reflexión para la construcción del conocimiento, mediante la creación de entornos de interacción social a través de herramientas para el trabajo colaborativo en red (Avello y Duart, 2016, párr.7).

Las tecnologías de la información y la comunicación permiten involucrar al alumno de manera directa en ese proceso de transformación que desarrolla su capacidad para la búsqueda, selección y reflexión de la información, pero estas competencias no se generan de manera automática, solo con conocer de computación e informática, tampoco conectándose a la web o dominando las redes sociales.

Por tanto, para aplicar los nuevos medios de comunicación a la educación se debe aprender a usarlos adecuadamente, cualquier programa educativo basado en estos recursos, debería considerar que no todos los alumnos tienen el mismo nivel de competencia comunicativa y de uso, investigación y análisis de la información de los medios.

En la enseñanza, los nuevos medios influyen, en los siguientes aspectos curriculares: en la nueva formación y actividad de los docentes, en la redefinición de los lugares formativos, en los enfoques didácticos y en las estrategias de comunicación educativa, en la organización del centro escolar y en el papel que debe asumir el alumnado (Lacruz, 2002, p.13).

Cabe mencionar, que el hecho de que el alumno sea más independiente y autónomo no quiere decir que sea autodidacta durante todo el proceso de enseñanza aprendizaje. Podría creerse que con el uso de los medios, el docente pierde acción en el proceso, y pensar esto representa un grave peligro en la educación, pues, el docente es factor clave, debe entenderse que cambia sus actividades, pero no quiere decir que, pierde importancia, y que debe permanecer ausente, mantenerse al margen y alejado del alumno.

Es importante que el docente se encuentre preparado y sea competente en la tecnología, pues es necesaria su contextualización para lograr involucrar al alumno y adaptar sus programas educativos a los nuevos escenarios que se le presenten.

Debe comprender los cambios a los que se enfrenta para poder transmitirlo al estudiante. Y no solamente que los docentes sean competentes en la tecnología, sino que además

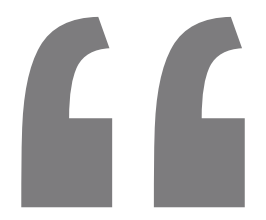

El usuario ha

pasado a tener

un papel muy

dinámico, a dar a

conocer su propia

voz, sus ideas y sus

obras en la web

y a relacionarse

con otros usuarios

a un nivel sin

precedentes en la

historia". 
puedan orientar a los alumnos a enfrentarse a los medios de manera correcta y consciente, haciendo uso correcto de la información para protegerlos de los peligros y amenazas que encierra la tecnología, dándole un uso que permita desarrollar la inclusión de éstas de modo significativo al ámbito educativo (Dussel y Quevedo, 2010, p.42).

Es necesario por ello, innovar en lo que corresponde a las estrategias pedagógicas, generar nuevas maneras de enseñar, para lograr aprendizajes significativos en estos nuevos contextos. "La innovación pedagógica es la voluntad de cambio, planeada y puesta en práctica para mejorar la praxis de la docencia (enseñanzaaprendizaje) mediante la flexibilidad, documentación y sistematización analítica para acometer cambios, mejoras, actualizaciones, nuevas teorías y conocimientos aplicados" (Roldán, 2013, p.2).

Es importante que el docente se adapte a estos nuevos escenarios, para crear procesos de enseñanzaaprendizaje innovadores. "La actividad docente debe ser consciente de esta realidad y adaptar o crear métodos que permitan utilizar los servicios TIC como herramientas para el desarrollo profesional y personal del alumno" (Blanco, 2009, p.160).

El diseño instruccional, por ejemplo, es básico para estructurar un plan adecuado con una metodología que incluya actividades con objetivos enfocados en la autonomía del alumno y su participación activa, a partir de una alfabetización mediática que permita el desarrollo de su capacidad de búsqueda, análisis y reflexión de la información con una actitud crítica, poniendo en práctica también su competencia comunicativa, adaptado siempre, claro está, a la materia que se imparte, involucrando todos los medios necesarios para el desarrollo integral del alumno.

"El diseño instruccional, en el ámbito educativo, debe facilitar el procesamiento significativo de la información y del aprendizaje; por tanto, ha de ser capaz de enseñar el conocimiento organizadamente" (Martínez, 2009, p.110).

La clave del éxito en el uso de los nuevos medios de comunicación en la educación se encuentra principalmente en la innovación pedagógica que incluya un diseño instruccional y una selección adecuada de los medios, tomando en cuenta que el docente debe estar preparado para ello y que el alumno tiene la madurez y el nivel requerido para enfrentarse a las nuevas tecnologías de la educación.

"Es necesario desarrollar en los jóvenes la madurez suficiente para identificar y valorar los efectos que las tecnologías de la comunicación tienen en las sociedades" (Cervera, 2010, p.106).

"Es importante buscar nuevas formas de aplicación en la enseñanza, implicando la movilización de una diversidad de estrategias y metodologías docentes que favorezcan una enseñanza activa, participativa, colaborativa y constructiva que lleve al estudiante a ser un verdadero agente en su proceso de enseñanza-aprendizaje" (Cabero, 2015, p.24). El uso y aplicación de los nuevos medios en la educación no debe ser una acción improvisada, al contrario, requiere de un mayor esfuerzo para no pasar por alto ningún detalle, hay muchos puntos que deben considerarse para un buen desarrollo de los programas educativos y exige preparación, contextualización, comunicación y adaptación.

La tecnología ha generado procesos evolutivos en la educación, que provocan cambios en las metodologías y estrategias de enseñanza-aprendizaje, con ello, el rol de los actores del proceso también ha sufrido cambios y es necesaria una adaptación de los mismos a los nuevos escenarios

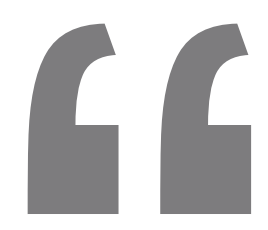

Los nuevos medios de comunicación a la educación se debe aprender a usarlos adecuadamente". 


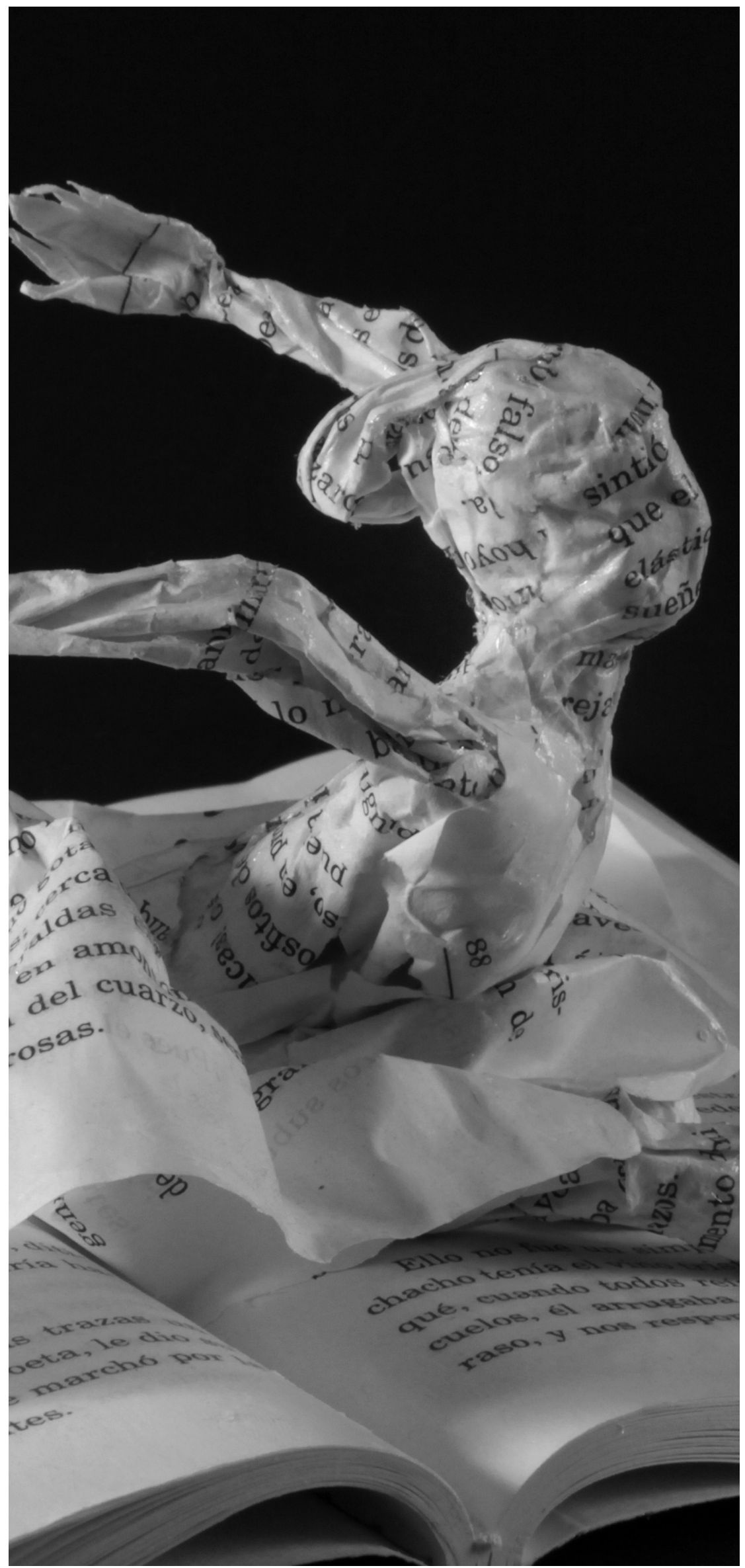

educativos. Las tecnologías de la información y la comunicación le dan a la educación diversos caminos para recorrer y es posible obtener resultados óptimos, pero la introducción de los mismos debe tomarse de manera seria, por parte, tanto del alumno como del docente, es necesario entender que el proceso de adaptación se da poco a poco, pero también tener la disposición para mantenerse actualizado y crear planes a partir de metodologías que favorezcan el crecimiento intelectual del alumno y el desarrollo de las competencias necesarias para enfrentarse a la realidad actual.

Es ahí donde debe surgir la innovación pedagógica, teniendo voluntad para el cambio y flexibilidad para la adaptación, siendo conscientes de que el mundo actual lo exige y más que ser una tendencia, es una necesidad que se debe cubrir en nuestra sociedad. No es una tarea fácil, pero es una misión trascendental y por ello, vale la pena luchar, enfrentarse e involucrarse en los procesos de transformación de la educación, para llegar así a las metas establecidas, siendo altamente competentes en el ámbito social y laboral.

En la educación actual, una frase debe estar siempre presente en el docente: un punto es dominar la tecnología y otro, muy distante, es saber educar con la tecnología. 


\section{Referencias}

Avello, R. y Duart, J. (2016). Nuevas tendencias de aprendizaje colaborativo en e-learning. Claves para su implementación efectiva. Revista SciELO. Estudios pedagógicos. Vol. 42 No.1. versión On-line ISSN 0718-0705. Disponible en: http://dx.doi.org/10.4067/S071807052016000100017.

Blanco, A. (2009). Desarrollo y Evaluación de Competencias en Educacion Superior. Narcea Ediciones.

Bustos, A. (2005). Estrategias Didácticas para el uso de las TIC's en la Docencia Universitaria Presencial. Barcelona: Pontificia Universidad Católica de Valparaíso.

Buxarrais, M. y Ovide, E. (2011). El impacto de las nuevas tecnologías en la educación en valores del siglo XXI. Revista SciELO. ISSN 1665-109X. No. 37. Disponible en: http://www. scielo.org.mx/scielo.php?script=sci_arttext\&pid=\$1665-109X2011000200002.

Cabero, J. (2015). Reflexiones educativas sobre las tecnologías de la información y la comunicación (TIC). Revista TCyE Tecnología, Ciencia y Educación. ISSN:2444-250X / ISSN-e:2444-2887. CEF, núm. 1 (mayo-agosto 2015, págs.19-27). Disponible en: https://idus. us.es/xmlui/bitstream/handle/11441/32285/Reflexiones_educativas_sobre_las_Tecnolo. pdf?sequence=1\&isAllowed=y.

Cervera, D. (2010). Tecnología. Complementos de formación disciplinar. Barcelona: Editorial Grao.

Dussel I. y Quevedo L. (2010). Educación y nuevas tecnologías: los desafíos pedagógicos ante el mundo digital. Buenos Aires: Santillana.

Lacruz, M. (2002). Nuevas tecnologías para futuros docentes. La mancha: Universidad de Castilla-La Mancha.

Martínez, A. (2009). El diseño instruccional en la educación a distancia: un acercamiento a los modelos apertura. Redalyc. Vol. 9. No.10. Disponible en: http://www.redalyc.org/ pdf/688/68812679010.pdf.

Roldán, N. (2013). Innovaciones pedagógicas y TIC: retos para una sola educación. Revista Virtual Universidad Católica del Norte. ISSN-0124-5821. No. 39 (Mayo-Agosto, 2013). Disponible en: http://revistavirtual.ucn.edu.co/index.php/RevistaUCN/article/view/421/894.

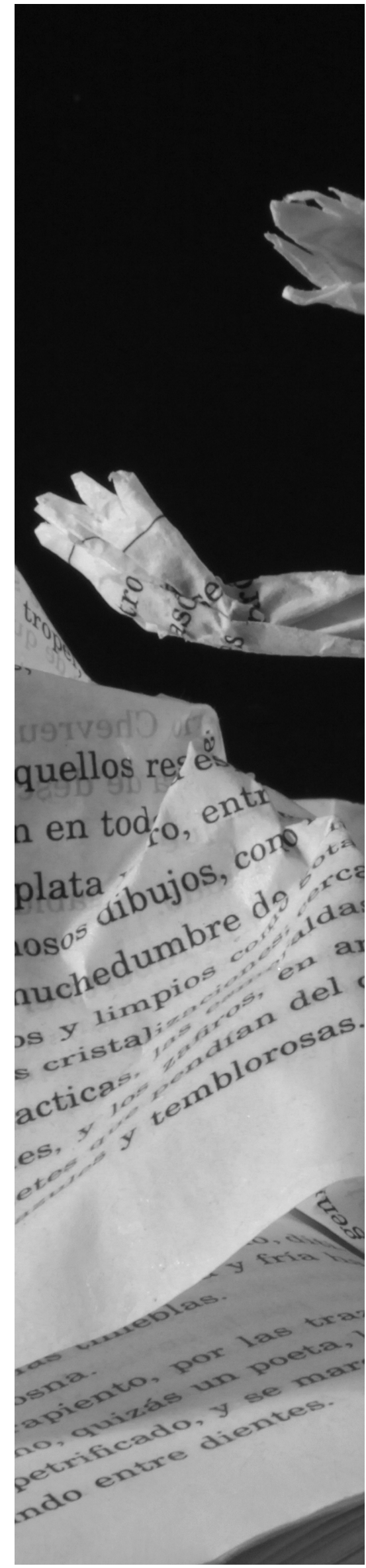




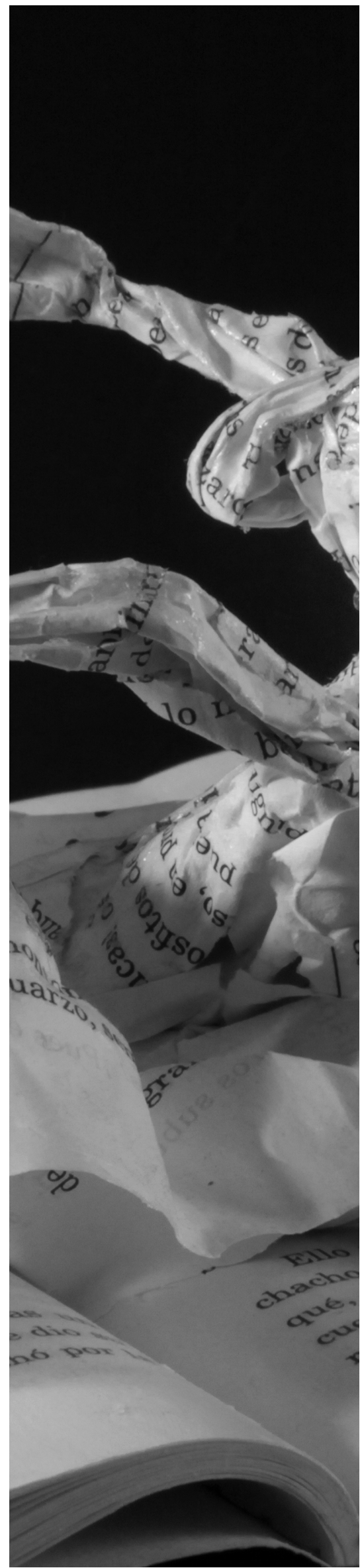

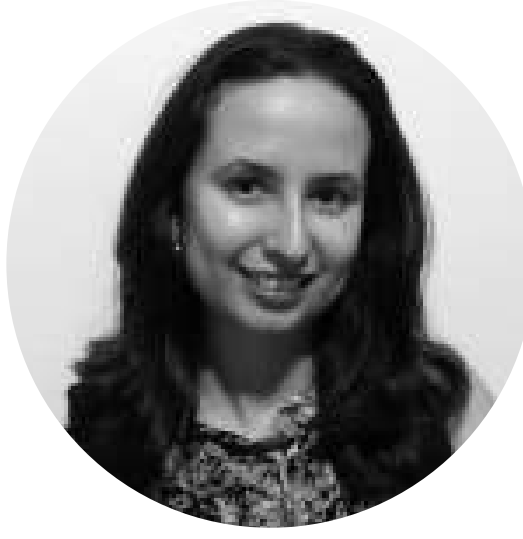

Sandra Guadalupe Altamirano Galván

Licenciada en Artes Visuales con acentuación en Artes Gráficas, egresada de la Facultad de Artes Visuales de la UANL, cuenta con una Maestría en Ciencias de la Comunicación con acentuación en Nuevas Tecnologías de la UANL y concluyó sus estudios de Doctorado en Educación con acentuación en Comunicación y Tecnología Educativa. Se ha desarrollado en diversas áreas del diseño, tales como identidad corporativa, desarrollo de campañas publicitarias, fotografía, diseño editorial, entre otros. Su trabajo también se desarrolla en el área musical, como composición, grabación de jingles y radio. Actualmente es coordinadora de la Licenciatura en Diseño Gráfico de la Facultad de Artes Visuales, así también imparte clases del área editorial y de identidad en dicha Facultad.

Correo electrónico:

sandra.altamiranoglv@uanl.edu.mx
Recibido: 30/04/2017

Aceptado: 20/11/2017 